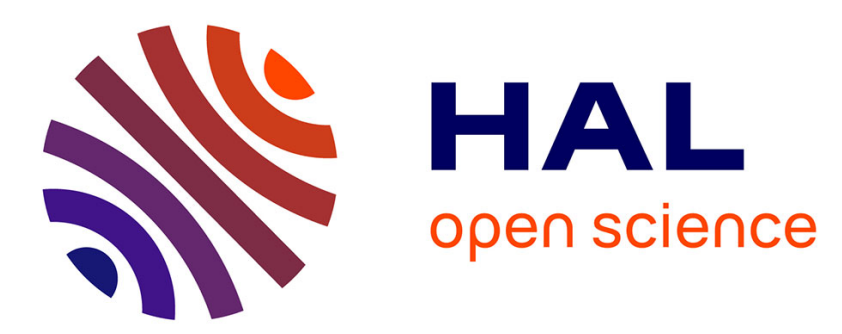

\title{
Apparent Viscosity of a Mixture of a Newtonian Fluid and Interacting Particles
}

Aline Lefebvre, Bertrand Maury, Aline Lefebvre-Lepot

\section{To cite this version:}

Aline Lefebvre, Bertrand Maury, Aline Lefebvre-Lepot. Apparent Viscosity of a Mixture of a Newtonian Fluid and Interacting Particles. Comptes Rendus Mécanique, 2005, 333 (12), pp.923-933. hal-00728382

\section{HAL Id: hal-00728382 \\ https://hal.science/hal-00728382}

Submitted on 5 Sep 2012

HAL is a multi-disciplinary open access archive for the deposit and dissemination of scientific research documents, whether they are published or not. The documents may come from teaching and research institutions in France or abroad, or from public or private research centers.
L'archive ouverte pluridisciplinaire HAL, est destinée au dépôt et à la diffusion de documents scientifiques de niveau recherche, publiés ou non, émanant des établissements d'enseignement et de recherche français ou étrangers, des laboratoires publics ou privés. 


\title{
Apparent Viscosity of a Mixture of a Newtonian Fluid and Interacting Particles
}

\author{
Aline Lefebvre*and Bertrand Maury ${ }^{\dagger}$
}

September 8, 2012

\begin{abstract}
We investigate the behaviour of fluid-particle mixtures subject to shear stress, by mean of direct simulation. This approach is meant to give some hints to explain the influence of interacting red cells on the global behaviour of the blood. We concentrate on the apparent viscosity, which we define as a macroscopic quantity which characterizes the resistance of a mixture against externally imposed shear motion. Our main purpose is to explain the non-monotonous variations of this apparent viscosity when a mixture of fluid and interacting particles is submitted to shear stress during a certain time interval. Our analysis of these variations is based on preliminary theoretical remarks, and some computations for some wellchosen static configurations.
\end{abstract}

\section{Introduction}

The viscosity of a diluted suspension can be estimated from the exact solution to the Stokes equations for a single particle in an infinite fluid domain (this approach dates back to Einstein [5] in 1906). In many situations, e.g. red cells in the blood, the dilution asumption is no longer valid, and inclusions are likely to interact with each other in a complex way. Under some assumptions the behaviour of neighbouring particles can be described by mean of analytic expressions or asymptotic development. See e.g. [2] where the motion of two spheres in a shear flow is described. More recently, Stokesian Dynamics has been applied to compute the motion of spheres in a linear Stokes flow for particular geometries (see [4], [8]). But the overall behaviour of nonhomogeneous, many-body mixtures under general shear conditions calls for the use of direct numerical methods.

As a consequence, direct simulation of fluid-particle mixtures motivated a great amount of research during the last decade. Some authors, like Glowinski (see $[6,7]$ ) use a cartesian mesh which covers the overall computational domain,

*Laboratoire de Mathématiques, Université Paris-Sud

†Laboratoire de Mathématiques, Université Paris-Sud 
and the rigid motion is taken into account by a Lagrange multiplier. The other class of approaches relies on a moving mesh which follows the geometry of the fluid domain. This approach has been followed by [10], [9], and [13].

The present work is based on this second approach, which we think is more adapted to shear flow of highly concentrated suspension, as the presence of a (possibly) fine mesh which covers interparticle gaps makes it possible to compute accurately in these high stress regions. Moreover, integrating the degrees of freedom for the particles into the finite element space allows to compute highly viscous flows with unconditional stability, whereas other methods which decouple fluid and particles are more adapted to situations where inertia (at least inertia of the rigid bodies) plays a significant role. Although the numerical approach we follow would make it possible to handle Navier-Stokes flows and general geometries, we chose to limit ourselves here to Stokes flow, in order to show how a globally nonlinear behaviour can be recovered even though the instantaneous fluid model is itself linear.

\section{Continuous model}

We consider a rectangular domain $\Omega$ filled with a mixture of a Newtonian fluid and $N$ rigid particles. The viscosity of the fluid is denoted by $\mu$. All particles are circular, and their common radius is $r$. We denote by $\mathbf{F}_{i}$ the force exerted on particle $i$. We shall consider the situation where $\mathbf{F}_{i}$ is a sum of forces exerted by the other particles. The mixture domain $\Omega$ is a rectangle $2 a \times L$ (see figure 1). The flow is periodic in the $y$-direction. Left and right walls are supposed to move vertically with velocities $-U_{0} \mathbf{e}_{y}$ and $+U_{0} \mathbf{e}_{y}$, respectively. Origin of the reference frame $(x, y)$ is set on the centerline.

Let $\mathbf{X}_{i}=\mathbf{X}_{i}(t)$ be the center of particle $i$. We denote the fluid domain by

$$
\Omega_{F}(t)=\Omega \backslash \bigcup_{i=1}^{N} \overline{B\left(\mathbf{X}_{i}(t), r\right)} .
$$

The surrounding fluid is supposed to obey the incompressible Stokes equations in the moving fluid domain $\Omega_{F}(t)$,

$$
\left\{\begin{aligned}
-\mu \Delta \mathbf{u}+\nabla \mathrm{p} & =0 \\
\nabla \cdot \mathbf{u} & =0
\end{aligned}\right.
$$

while equilibrium of the particles at each time gives

$$
\left\{\begin{array}{l}
\int_{\Gamma_{i}} \sigma \cdot \mathbf{n}=\mathbf{F}_{i}, \\
\int_{\Gamma_{i}} \mathbf{r}_{i} \times \sigma \cdot \mathbf{n}=0,
\end{array}\right.
$$

where $\mathbf{r}_{i}$ is the position vector relatively to the center $\mathbf{X}_{i}$ of particle $i$, and $\sigma$ the stress tensor $\left(I_{d}\right.$ is the identity matrix)

$$
\sigma=\mu\left(\nabla \mathbf{u}+{ }^{t} \nabla \mathbf{u}\right)-\mathrm{pI}_{\mathrm{d}} .
$$


The no-slip conditions on the particles surface (denoted by $\Gamma_{i}(t)$ for the $i$-th particle) are

$$
\mathbf{u}(\mathbf{x})=\mathbf{V}_{i}+\omega_{i} \times \mathbf{r}_{i} \text { on } \Gamma_{i} \text { for } 1 \leq i \leq N,
$$

where $\mathbf{V}_{i}$ is the translational velocity of particle $i$ and $w_{i}$ is the angular velocity of particle $i$.
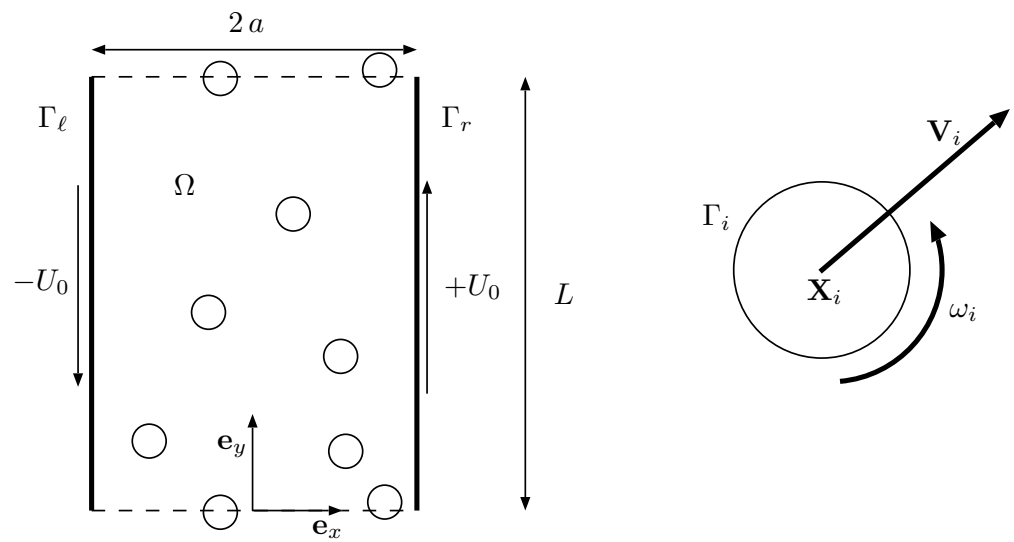

Figure 1 : Notations. Notations.

We shall denote by $\mathbf{V}=\left(\mathbf{V}_{1}, \ldots, \mathbf{V}_{N}\right)$ and $\omega=\left(\omega_{1}, \ldots, \omega_{N}\right)$ the vectors corresponding to the translational and rotational degrees of freedom. Let $\mathbf{X}=\left(\mathbf{X}_{1}, \ldots, \mathbf{X}_{N}\right)$ be given. Denoting by $H_{\sharp}^{1}\left(\Omega_{F}\right)$ the set of all those fields in $H^{1}\left(\Omega_{F}\right)$ which are periodic in the vertical direction, we introduce the space

$$
\begin{gathered}
\Pi=\left\{\mathbf{U}=(\mathbf{u}, \mathbf{V}, \omega)=\left(\mathbf{u}, \mathbf{V}_{1}, \ldots, \mathbf{V}_{N}, \omega_{1}, \ldots, \omega_{N}\right) \text { such that } \mathbf{u} \in H_{\sharp}^{1}\left(\Omega_{F}\right)^{2}, \mathbf{V}_{i} \in \mathbb{R}^{2}, w_{i} \in \mathbb{R},\right. \\
\left.\mathbf{u}(x)=\mathbf{V}_{i}+w_{i} \times \mathbf{r}_{i} \text { on } \Gamma_{i},\left.\mathbf{u}\right|_{\Gamma_{\ell}}=-U_{0} \mathbf{e}_{y},\left.\mathbf{u}\right|_{\Gamma_{r}}=U_{0} \mathbf{e}_{y}\right\}
\end{gathered}
$$

and its homogeneous counterparts $\Pi_{0}$ (space $\Pi$ for $\left.U_{0}=0\right)$. The instantaneous variational formulation reads

$\left\{\begin{array}{l}\text { Find } \mathbf{U}=(\mathbf{u}, \mathbf{V}, \omega) \in \Pi \text { and } \mathrm{p} \in L_{0}^{2}\left(\Omega_{F}\right) \text { such that : } \\ \frac{\mu}{2} \int_{\Omega_{F}}\left(\nabla \mathbf{u}+{ }^{t} \nabla \mathbf{u}\right):\left(\nabla \tilde{\mathbf{u}}+{ }^{t} \nabla \tilde{\mathbf{u}}\right)-\int_{\Omega_{F}} \mathrm{p} \nabla \cdot \tilde{\mathbf{u}}-\sum_{i=1}^{N} \tilde{\mathbf{V}}_{i} \cdot \mathbf{F}_{i}=0, \quad \forall \tilde{\mathbf{U}}=(\tilde{\mathbf{u}}, \tilde{\mathbf{V}}, \tilde{\omega}) \in \Pi_{0} \\ \int_{\Omega_{F}} \tilde{\mathrm{p}} \nabla \cdot \mathbf{u}=0 \quad \forall \tilde{\mathrm{p}} \in L_{0}^{2}\left(\Omega_{F}\right)\end{array}\right.$

where $L_{0}^{2}\left(\Omega_{F}\right)$ is the set of $L^{2}$ functions with zero mean value over $\Omega_{F}$.

The velocity field $\mathbf{U}=(\mathbf{u}, \mathbf{V}, \omega)$ is also the unique solution to the following constrained minimization problem:

$$
\left\{\begin{array}{l}
\operatorname{Minimize} J(\tilde{\mathbf{U}})=\frac{\mu}{4} \int_{\Omega_{F}}\left|\nabla \tilde{\mathbf{u}}+{ }^{t} \nabla \tilde{\mathbf{u}}\right|^{2}-\sum_{i=1}^{N} \tilde{\mathbf{V}}_{i} \cdot \mathbf{F}_{i} \\
\text { over } \Pi_{\text {div }}=\left\{\tilde{\mathbf{U}}=(\tilde{\mathbf{u}}, \mathbf{V}, \tilde{\omega}) \in \Pi \text { such that } \nabla \cdot \tilde{\mathbf{u}}=0 \in \Omega_{F}\right\}
\end{array}\right.
$$


Note that, as we neglect inertia, the evolution problem for the particles can be written as a system of ordinary differential equations: $\dot{\mathbf{X}}=\mathbf{V}(\mathbf{X})$, where the mapping $\mathbf{X} \mapsto \mathbf{V}(\mathbf{X})$ is the particle part of the solution to problem (5).

\subsection{Apparent viscosity}

The notion of apparent viscosity on which we shall base our approach corresponds to what is actually measured by most viscosimeters. It is defined as follows: we consider the pure Couette flow of an homogeneous fluid of viscosity $\tilde{\mu}$ under the same conditions. For a certain value of $\tilde{\mu}$, the vertical forces exerted on the walls are identical to the forces exerted by the actual mixture. The apparent viscosity is defined as this very value.

The vertical components of those forces exerted on the walls are :

$$
F_{l}=\int_{\Gamma_{\ell}} \mathbf{e}_{y} \cdot \sigma \cdot \mathbf{e}_{x} \text { and } F_{r}=\int_{\Gamma_{r}} \mathbf{e}_{y} \cdot \sigma \cdot\left(-\mathbf{e}_{x}\right) .
$$

In case of a newtonian fluid, the exact solution $(\mathbf{u}, \mathrm{p})$ being

$$
\left\{\begin{array}{l}
\mathbf{u}(x, y)=U_{0} \frac{x}{a} \mathbf{e}_{y} \\
\mathrm{p}(x, y) \equiv 0
\end{array}\right.
$$

we have $F_{0}=F_{\ell}-F_{r}=2 \mu \frac{U_{0}}{a} L$. The apparent viscosity can therefore be expressed as

$$
\mu_{\text {app }}=\frac{a}{2 L U_{0}} F_{0} \text { where } F_{0}=\int_{\Gamma_{\ell} \cup \Gamma_{r}} \mathbf{e}_{y} \cdot \sigma \cdot \mathbf{e}_{x} .
$$

\section{Theoretical considerations}

We summarize here some simple properties of the apparent viscosity which are direct consequences of its definition.

\subsection{Passive particles}

We suppose in this section that there are no forces exerted on the particles. In this case, there is an equivalent expression for the apparent viscosity, which relates to dissipated energy. We multiply momentum equation (1) by $\mathbf{u}$ and integrate it over $\Omega_{F}$. Integration by parts gives

$$
\frac{\mu}{2} \int_{\Omega_{F}}\left|\nabla \mathbf{u}+{ }^{t} \nabla \mathbf{u}\right|^{2}=\int_{\partial \Omega_{F}} \mathbf{u} \cdot(\sigma \cdot \mathbf{n})=U_{0} F_{0},
$$

so that

$$
\mu_{\mathrm{app}}=\frac{\mu a}{4 L U_{0}^{2}} \int_{\Omega_{F}}\left|\nabla \mathbf{u}+{ }^{t} \nabla \mathbf{u}\right|^{2},
$$


which is proportional to the functional which $\mathbf{u}$ minimizes (see formulation (6)). As a consequence, if we consider two sets of particles, the second one being larger in the sense that it covers the first one, there are obviously more constraints in the second one, and the minimum of the functionnal is larger. Finally, in accordance with intuition, if there are no forces, apparent viscosity increases when particules are added. As we shall see, it does not mean that the apparent viscosity is an increasing function of the solid fraction.

\section{$3.2 \quad$ Non-zero forces}

These considerations are no longer valid if we consider a system of particles submitted to forces. In that case, apparent viscosity may either increase or decrease with the intensity of the forces. Indeed, the energy balance now takes into account the action of these forces and the apparent viscosity expresses

$$
\mu_{\mathrm{app}}=\frac{\mu a}{4 L U_{0}^{2}} \int_{\Omega_{F}}\left|\nabla \mathbf{u}+{ }^{t} \nabla \mathbf{u}\right|^{2}-\frac{a}{2 L U_{0}^{2}} \sum \mathbf{V}_{i} \cdot \mathbf{F}_{i} .
$$

In order to investigate the variations of this quantity with respect to $\mathbf{F}$ and $U_{0}$, we separate their respective contributions to the solution $\mathbf{U}$. Let us denote by $\mathbf{U}^{0}=\left(\mathbf{u}^{0}, \mathbf{V}^{0}, \omega^{0}\right)$ the solution to problem (5) with $\mathbf{F}=0$ and $U_{0}=1$, and by $\mathbf{u}^{F}=\left(\mathbf{u}^{F}, \mathbf{V}^{F}, \omega^{F}\right)$ the solution to the same problem with $U_{0}=0$. By linearity of the problem, it holds $\mathbf{U}=U_{0} \mathbf{U}^{0}+\mathbf{U}^{F}$. Using this expression for $\mathbf{U}$ as well as the variational formulations for $\mathbf{U}^{0}$ and $\mathbf{U}^{F}$, we obtain another expression for the apparent viscosity:

$$
\mu_{\mathrm{app}}=\frac{\mu a}{4 L} \int_{\Omega_{F}}\left|\nabla \mathbf{u}^{0}+{ }^{t} \nabla \mathbf{u}^{0}\right|^{2}-\frac{a}{2 L U_{0}} \sum \mathbf{V}_{i}^{0} \cdot \mathbf{F}_{i}=\mu^{0}-\frac{a}{2 L U_{0}} \sum \mathbf{V}_{i}^{0} \cdot \mathbf{F}_{i} .
$$

Note that $\mu^{0}$ is the apparent viscosity in the case $\mathbf{F}=0$. It does not depend on $U_{0}$.

The influence of interaction (or external) forces is expressed by the scalar product $\sum \mathbf{V}_{i}^{0} \cdot \mathbf{F}_{i}$, whose sign depends on the configuration only. Fig. 2 illustrates this alternative. We consider two particles submitted to an attractive force. In the first case (on the left in figure 2), the forces tend to increase the apparent viscosity, whereas they make it decrease in the other case. In some situations, the apparent viscosity might even become negative, as can be checked for the second configuration if we have the force modulus go to infinity.

Another direct consequence of expression (8) is that, when the forces tend to increase $\mu_{\mathrm{app}}$, then $\mu_{\mathrm{app}}$ is a decreasing function of $U_{0}$ (for a fixed force field F).

We shall finish this section by considering another situation where the dependence of the apparent viscosity with respect to attractive forces is predictable. Suppose all interparticle forces derive from a convex potential of their distances:

$$
\mathbf{F}_{i}=\sum_{j \neq i} \mathbf{F}_{j i}, \mathbf{F}_{j i}=-k \nabla \Psi\left(D_{i j}\right)
$$


Consider now a static, stable configuration, corresponding to an equilibrium of the system of particles in a quiescent fluid. This means that the configuration minimizes the global potential energy

$$
E=\frac{k}{2} \sum_{j \neq i} \Psi\left(D_{i j}\right),
$$

over the set of feasible configuration (i.e. configurations with no overlapping). In this situation, any feasible motion $\mathbf{V}$ (i.e. any motion which respects the nonoverlapping constraints) of the set of particles has non-negative scalar product with the gradient of $E$, i.e. $\mathbf{V} \cdot \mathbf{F} \leq 0$. As the actual velocity field $\mathbf{V}^{0}$ is itself necessarily feasible, we are in a situation where forces tend to increase the apparent viscosity.
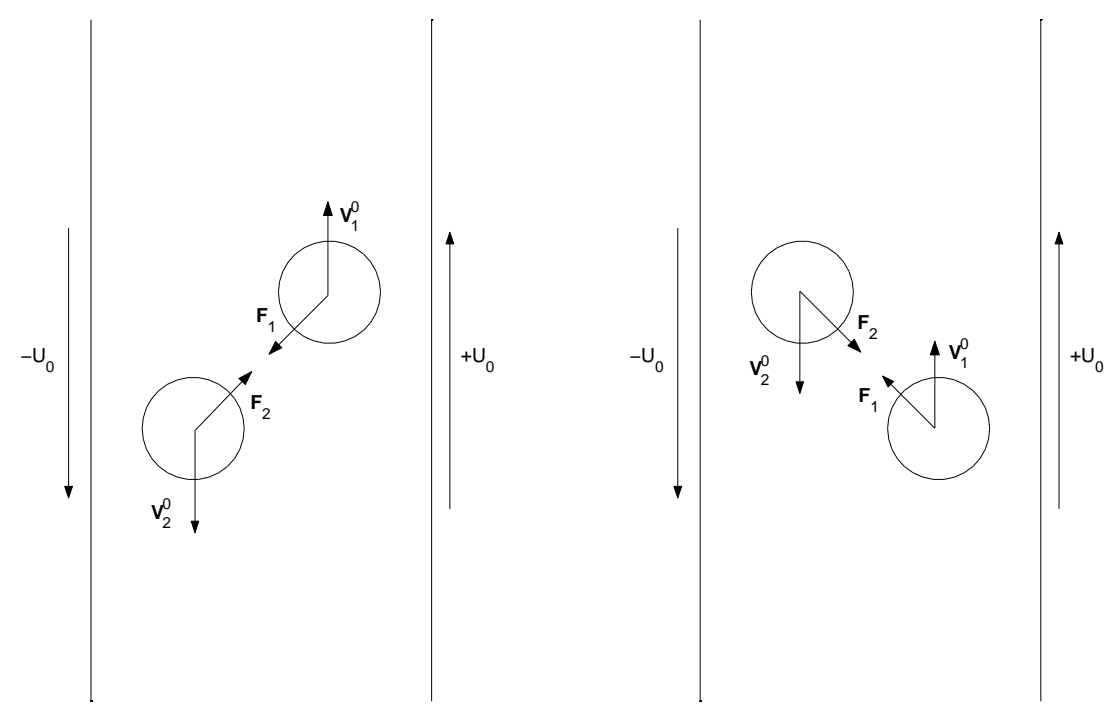

Figure 2: Opposite effects of an attractive force upon $\mu_{\text {app }}$ depending on the configuration. Effets opposés d'une force attractive sur $\mu_{\text {app }}$, selon la configuration.

\section{Numerical strategy}

Our approach is based on a direct discretization of space $\Pi$. It is thouroughly described in [13], and we shall simply indicate here the main features of the method we use:

1) the computation is performed on a mesh which is itself periodic, so that periodicity is taken into account in a strong way; 
2) a structured, thin, layer of elements is added around each particle, in order to ensure an accurate computation of lubrication forces. Furthermore, the global mesh is coarser far away from the particles, where the velocity gradients are expected to be smaller (see Fig. 3);

3) the degrees of freedom associated to the particles are integrated within the discretization space for the velocity, so that we end up with a standard symmetric positive definite stiffness matrix;

4) for non-stationary computation, as the fluid domain is likely to undergo large deformation, the mesh has to be regenerated from time to time. For Navier-Stokes flow, a projection of the velocity from the old mesh onto the new one is necessary. As for the computations which are presented here, this step is not necessary, because we neglect inertia.

We must add a few comments concerning the problem of particles getting close to each other. It is especially sensitive in the situation we consider, as we add attractive forces between particles, and those forces tend to put particles in contact. Different strategies have been used to overcome this problem. Some authors add a short-range repulsive force between neighbouring particles (see Glowinski [6]). Another strategy consists in refining the mesh in the neighbourhood of the interparticle gap (see $\mathrm{Hu}[9]$ ). The latter approach is justified by the following considerations, which applies to particles with a mass, and a fortiori to particles with no mass. Considering two smooth spheres in a viscous fluid, approaching each other at velocity $\dot{\varepsilon}$, where $\varepsilon$ is the distance, the lubrication force (which acts on both spheres with opposite directions) is known to be of order $\dot{\varepsilon} / \varepsilon$ (see Kim [11]). Therefore, if we denote by $F$ the modulus of a force which tend to approach them, the distance $\varepsilon$ obeys an equation like

$$
\ddot{\varepsilon}=-\frac{\dot{\varepsilon}}{\varepsilon}+F,
$$

so that $\varepsilon$ can be checked easily to remain positive, as soon as $F$ is bounded (or even locally integrable in time). Consequently, particles can be expected to avoid actual contact if the computation respects the physical model. Experiments suggest that the global behaviour of the mixture is not too sensitive to what is done at the local level, as far as reasonably diluted suspensions are concerned. Nevertheless, for high concentrations (say, solid fractions above 50\%), lubrication forces begin to play a crucial role in the overall behaviour. We chose here to use a fine mesh in the neighbourhood of the particles. Nevertheless, as interaction forces tend to put particles in contact, which would imply a breakdown of the computation (if particle boundary intersect, a new mesh cannot be generated), we perfom a projection-like step which ensures that interparticle distances are larger than a given parameter $\varepsilon$. This heuristic procedure is described in [13]. 


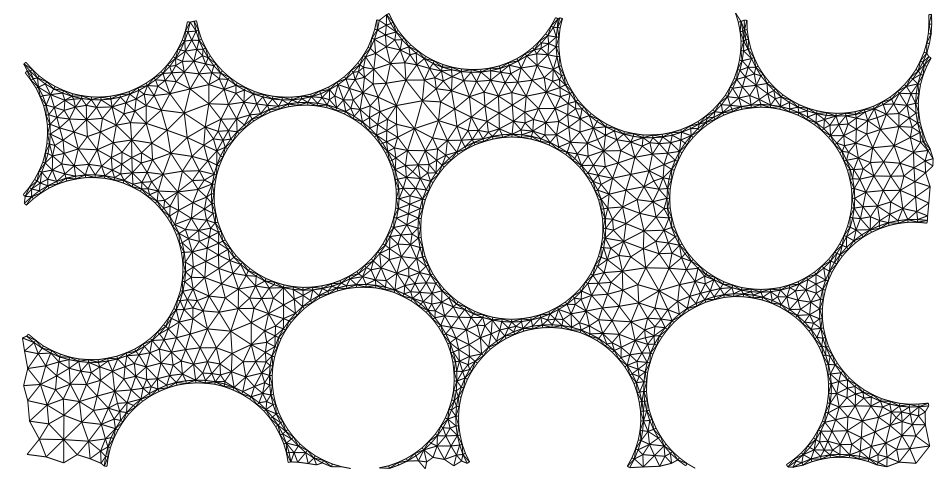

Figure 3 : Zoom of the mesh. Zoom du maillage.

\section{$5 \quad$ Numerical experiments}

\subsection{Passive particles}

This section presents results concerning the apparent viscosity of some mixtures when there is no interaction forces.

We first consider configurations with "uniform" distribution of the particles where the mimimal distance between the particles is bounded away from zero, and we plot apparent viscosity with respect to the solid fraction. By uniform distribution we mean the following: in a first step, particles centers are disposed randomly (according to a uniform law) in the computational domain, and a relative overlap (corresponding to $25 \%$ of the diameter) is tolerated, which means that if a new particle violates this condition, it is not created. In a second step, particles are swept away from each other according to the very same procedure which is used at each time step to ensure a minimal distance between particles. This correction can be thought of as a projection onto the set of feasible configurations, i.e. such that the minimal distance between particles is greater than a prescribed value $\varepsilon$. Note that a procedure based on a simple exclusion of any overlap excludes the creation of solid fractions above $45 \%$, whereas the acceptance of a certain amount of initial overlapping makes it possible to reach more than $60 \%$. 


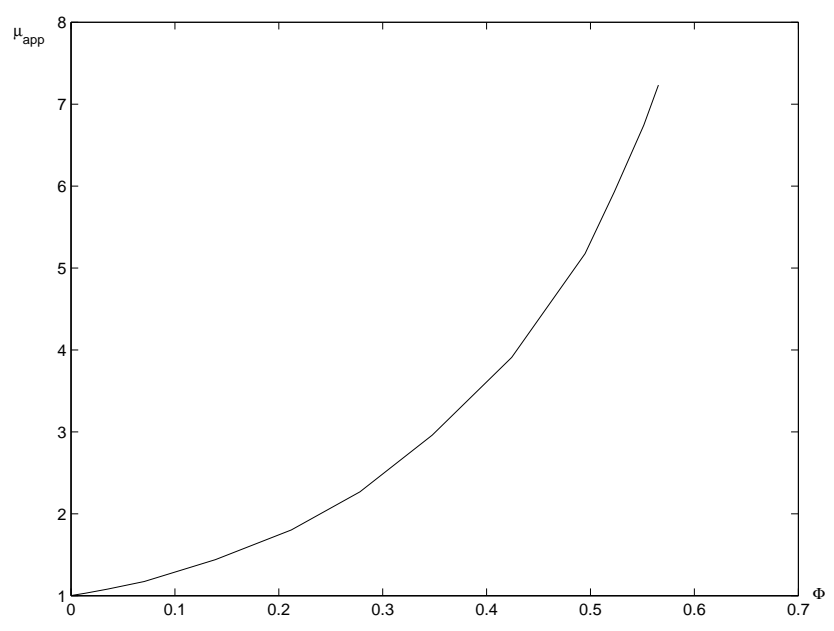

Figure 4 : Apparent viscosity with respect to solid fraction for uniform distributions. Viscosité apparente en fonction de la fraction solide pour une distribution uniforme.

We recover the fact that apparent viscosity increases with respect to solid fraction and tends to blow up as this fraction approaches its maximal value (see [1]). For higher solid fraction, the lubrication forces between particles play an increasing role, the dependance of $\mu_{\text {app }}$ upon solid fraction has to be investigated more carefully.

The next set of results illustrates the fact that apparent viscosity is not a monotonuous function of the solid fraction as soon as non-uniform distributions are considered. To that purpose, we considered non-uniform distributions with the same solid fraction $(25 \%)$, but with different overall distributions. We computed the apparent viscosity in 4 cases. Firstly, the distribution is uniform, in the sense indicated in the beginning of this section, and we find $\mu_{\text {app }}=2$. The second computation corresponds to an aggregate. The apparent viscosity is found to be greater (2.45), which illustrates the well-known fact that, in general, non-uniformity tends to increase the viscosity. The third computation indicates that the opposite behaviour is observed for some special types of aggregates. Indeed, when the particles are located in the neighbourhood of the centerline, the computed viscosity is found to be lower than in the two previous cases $\left(\mu_{\text {app }}=1.62\right)$, which can be explained by the presence of two "empty" zones in the neighbourhood of the walls. Note that the apparent viscosity would be almost the same if the cloud of particles were replaced by a rigid body covering the same zone. In this situation the apparent viscosity is therefore quite stable with respect to small perturbations of the particle distribution. In the 4th case, we built a bridge of particles between the two walls. The situation is now completely different, as the shear necessarily induces a deformation of the agglomerate of particles. These deformations are expensive from the energetic point of view because all particles are close to their neighbours, so that lubrication forces act against the deformation. In this case, the apparent viscosity 
is very sensitive to infinitesimal transformations of the configuration. We ran a 5 th computation by tuning up the minimal distance parameter to $15 \%$ of the particle radius (whereas it is $5 \%$ for configuration 4 ). We did not represent the configuration, as it cannot be distinguished from configuration 4 . The apparent viscosity dropped down to 3.47 (to be compared to 6.54 for the apparently identical situation). This type of situation has been investigated by Berlyand [3], from a theoretical point of view, but we are not yet able to perform quantitative comparisons between the computations and their results.
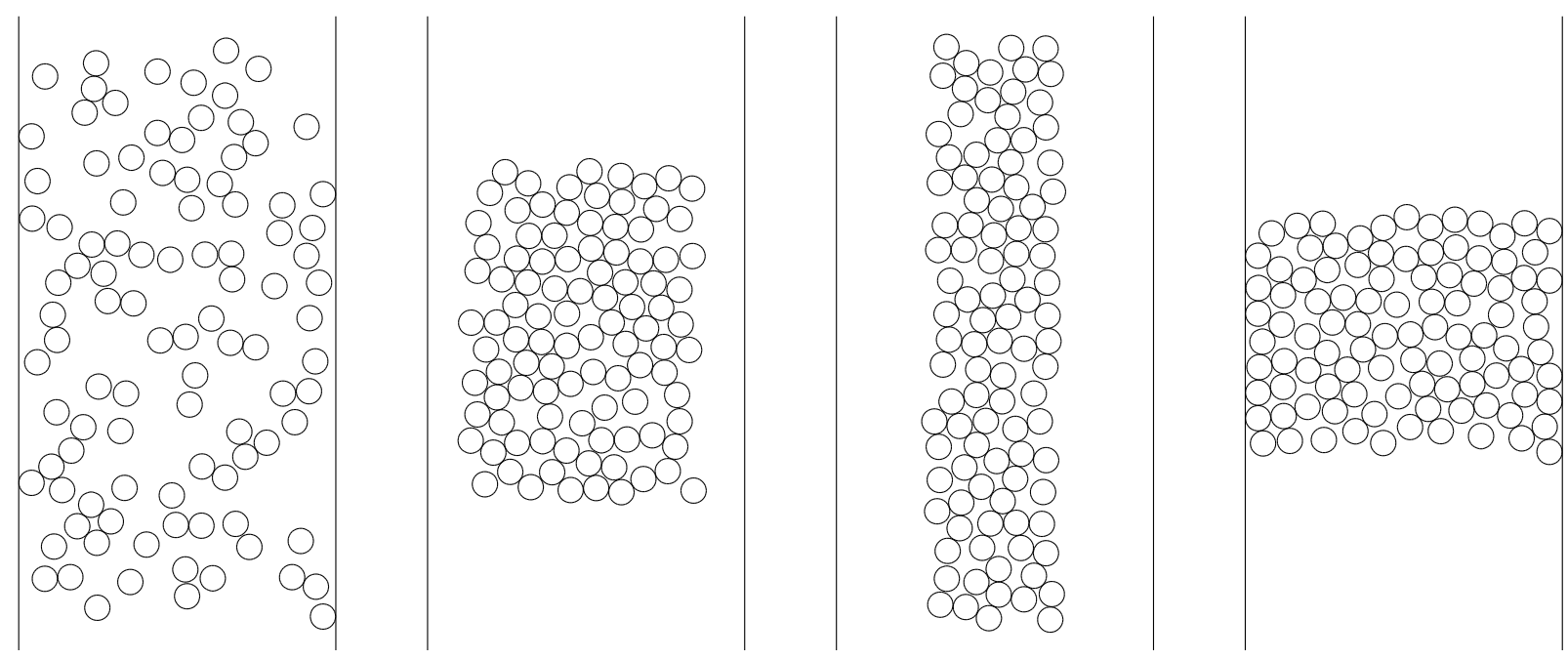

Figure 5 : Apparent viscosities 2.0, 2.45, 1.62, and 6.54. Viscosité apparente 2.0, 2.45, 1.62, et 6.54.

\subsection{Case with interaction forces}

We compute here the evolution of a mixture of fluid and 150 interacting particles. The interaction force between particles is attractive:

$$
\text { if } d_{i j} \leq 4 r \text { then } F_{i j}=\frac{0.1}{d_{i j}^{3}} \text { else } F_{i j}=0
$$

and geometrical parameters are $r=0.02, L=2, a=1 / 2$. The time step is chosen such that the largest displacement of a particle between two time iterates is $20 \%$ of the radius $r$. We prescribed the following shear history: at the beginning (from time label (1) to time label (2) on Fig. 6 ), $U_{0}=1$; then from (2) to (6), it is set to 50; and finally, from (6) to point (12), it is set back to 1 .

The first curve of figure 6 plots the apparent viscosity with respect to the time. We plotted a second curve to exhibit the part of geometrical parameters 
in the overall apparent viscosity. This second curve (dotted line) correspond to the apparent viscosity associated to the current configuration, as if there were no interaction forces. As previously, we shall denote this latter quantity by $\mu^{0}$ (see section 3.2). The configurations corresponding to time labels from (1) to (12) are represented in Figs. 7 and 8.

- ¿From (1) to (2), the configuration is close to a minimum of the potential energy. According to the remark which was made at the end of section 3.2, the apparent viscosity is greater than if there were no forces.

- ¿From (2) to (6), the velocity is much larger. As a consequence, the forces have a very small impact on the instantaneous apparent viscosity (see expression (8)), and $\mu_{\text {app }}$ is close to $\mu^{0}$. During this period of time, the aggregate is pulled apart by shear forces and the configuration becomes closer to the uniform distribution, which explains the fact that $\mu^{0}$ decreases (see figure 5 , cases 1 and 2), and so does $\mu_{\text {app }}$. The slight increase of both $\mu_{\text {app }}$ and $\mu^{0}$ between (5) and (6) can be explained by the fact that particles begin to reaggregate.

- ¿From (6) to (12), the velocity is set back to a smaller value, so that shear forces are dominated by attraction forces, and particules tend to aggregate again, which induces an overall increase of $\mu_{0}$. The behaviour of $\mu_{\text {app }}$ is more complicated, because of the effect which was illustrated by figure 2 . We can observe that $\mu_{\text {app }}$ begins to decrease and becomes lower than $\mu^{0}$ between (8) and (10), which means that the corresponding configurations are such that the scalar product in equation (8) is positive. Then $\mu_{\text {app }}$ increases while particles aggregate again and go closer to a minimum of the interaction energy.

\section{Conclusion}

We presented a numerical approach to investigate the complex dependence of the apparent viscosity of an active fluid-particle mixture upon geometrical distribution and interactions forces. The tool we developed provides what those simulations necessitate at a reasonable computational cost: an accurate description of close-range particle interactions, and the ability to deal with periodic geometries in order to perform long time simulations at constant solid fraction.

We show how non-monotonous variations of the apparent viscosity can be explained by a competition between geometrical effects (global properties of the particle distribution and local "closeness" of particles) and interactions forces. The approach we propose extends straightforwardly to more general situations: Navier-Stokes flows, domains with curved boundaries, non-circular particles. 


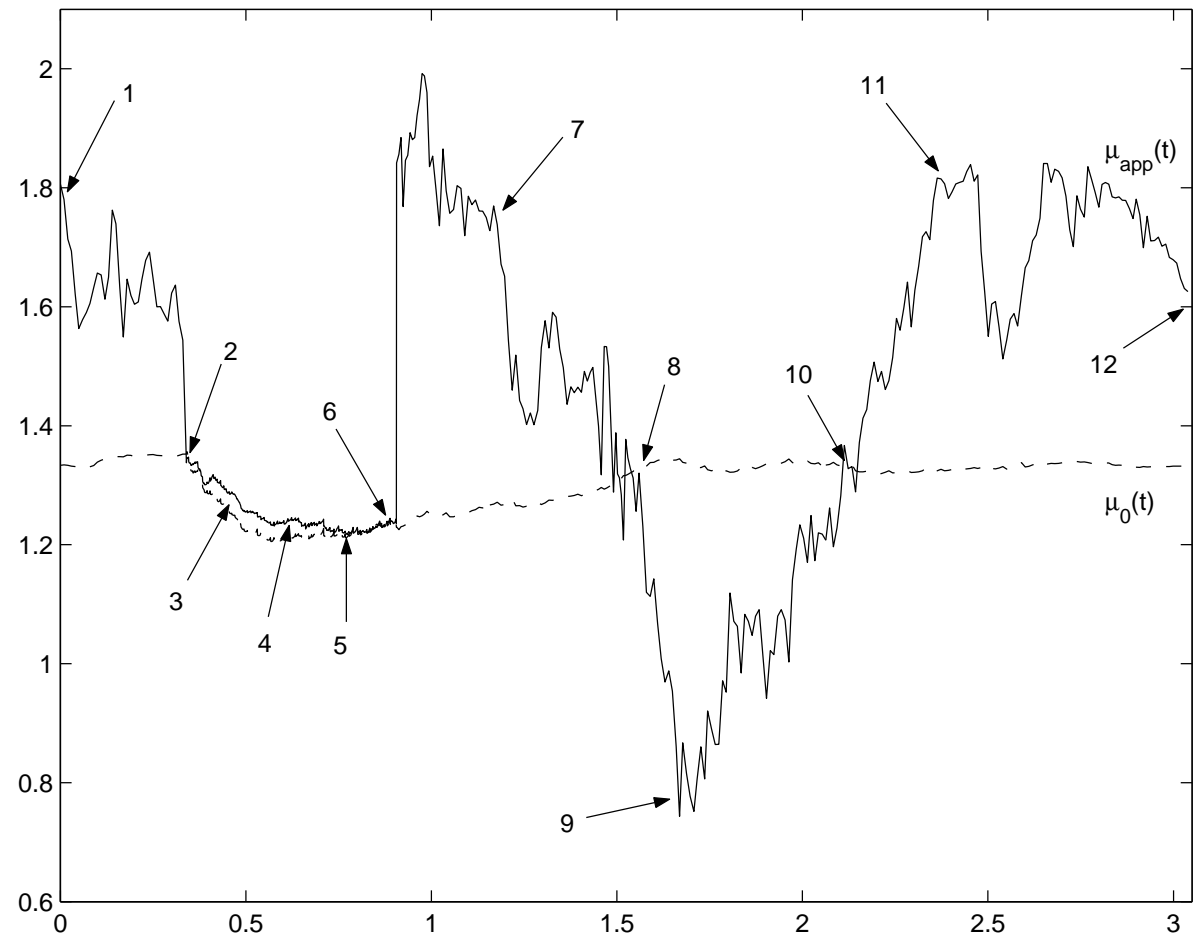

Figure 6 : Apparent viscosity versus time. Viscosité apparente en fonction du temps. 

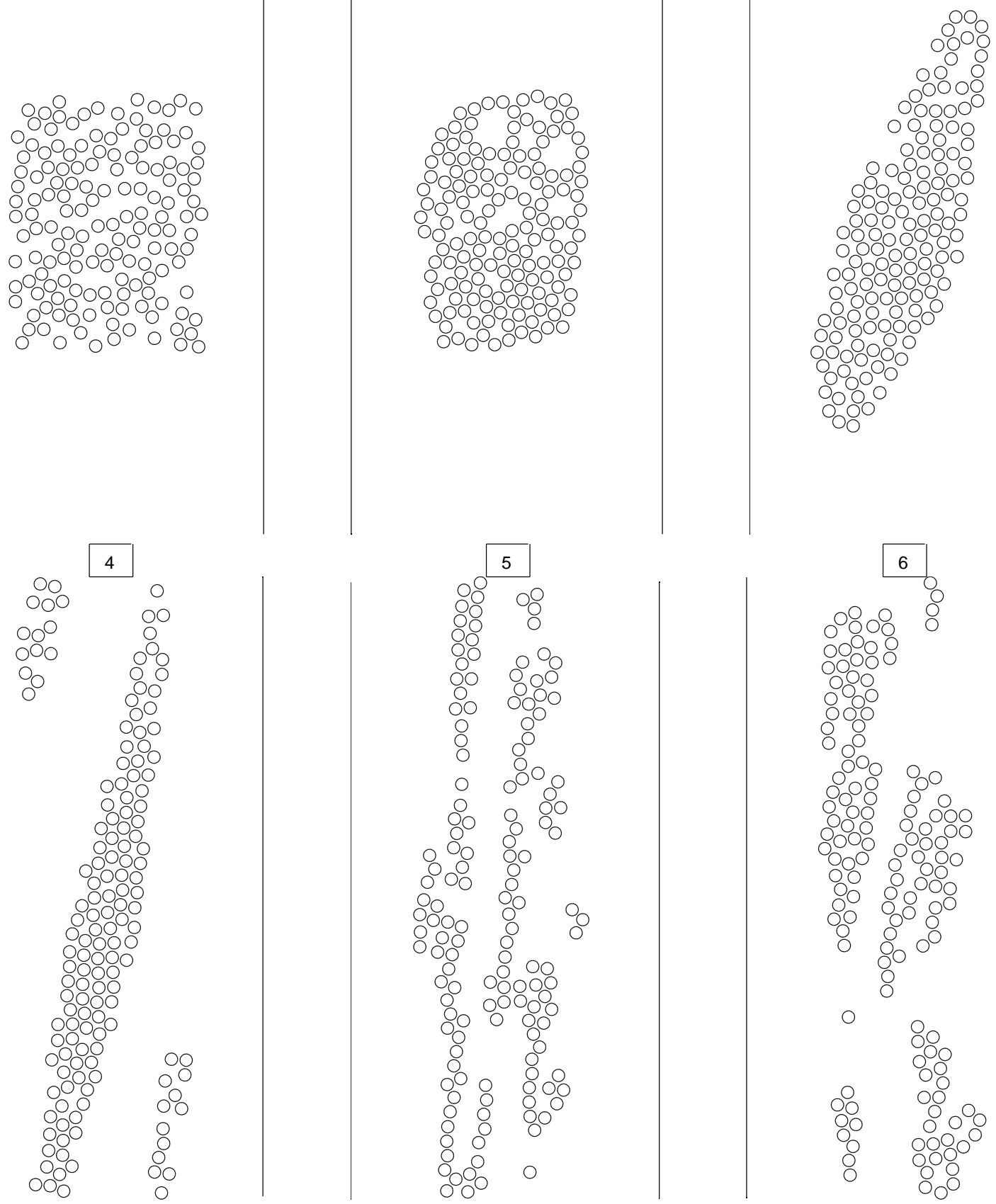

08088

0
0000
0000

000

80
0
0

00

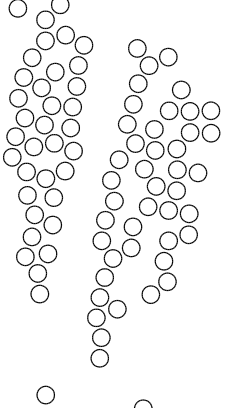

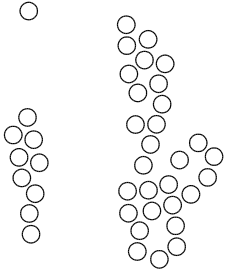


Figure 7 : Configurations (1) to (6). Configurations (1) à (6).

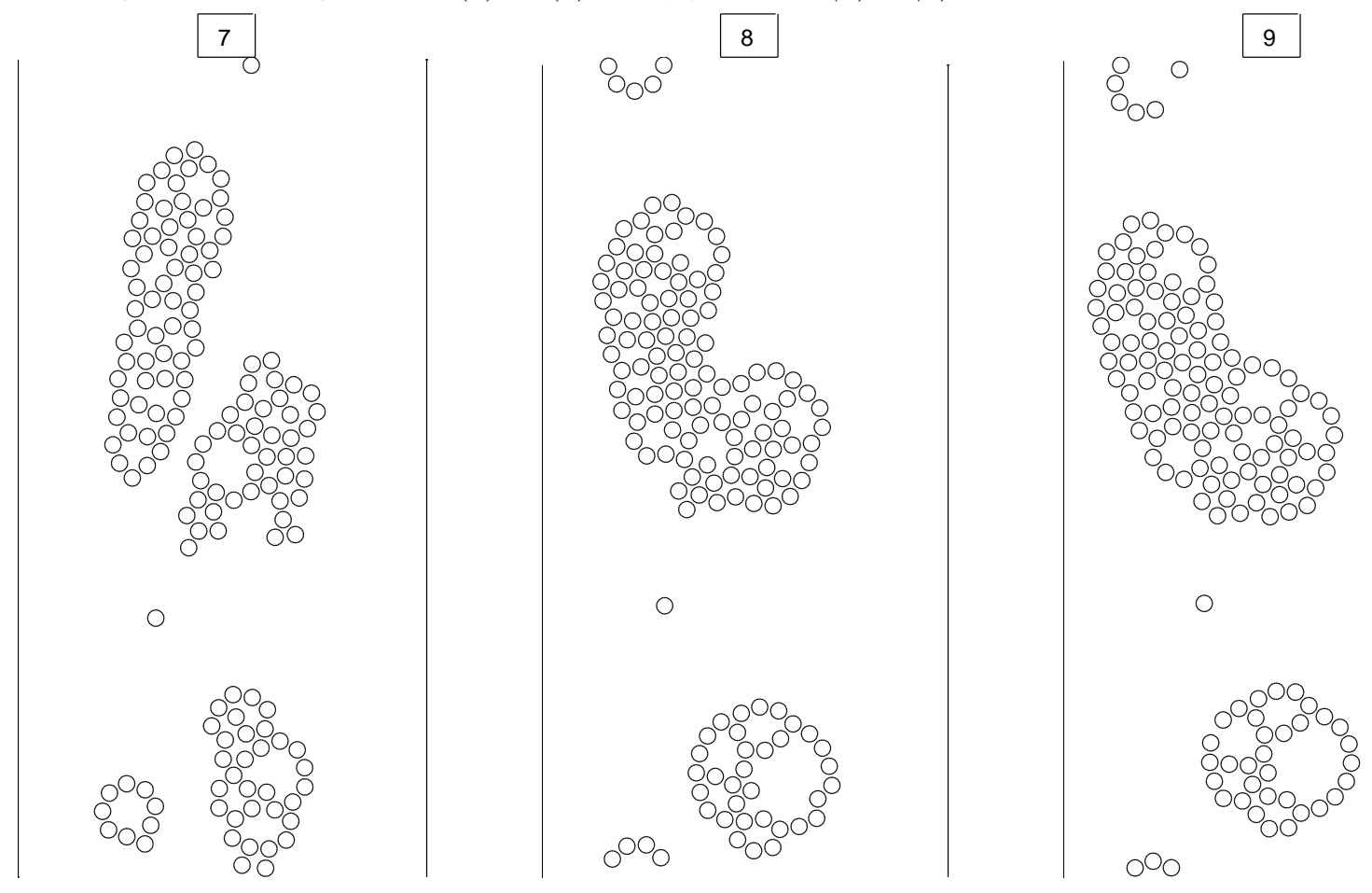



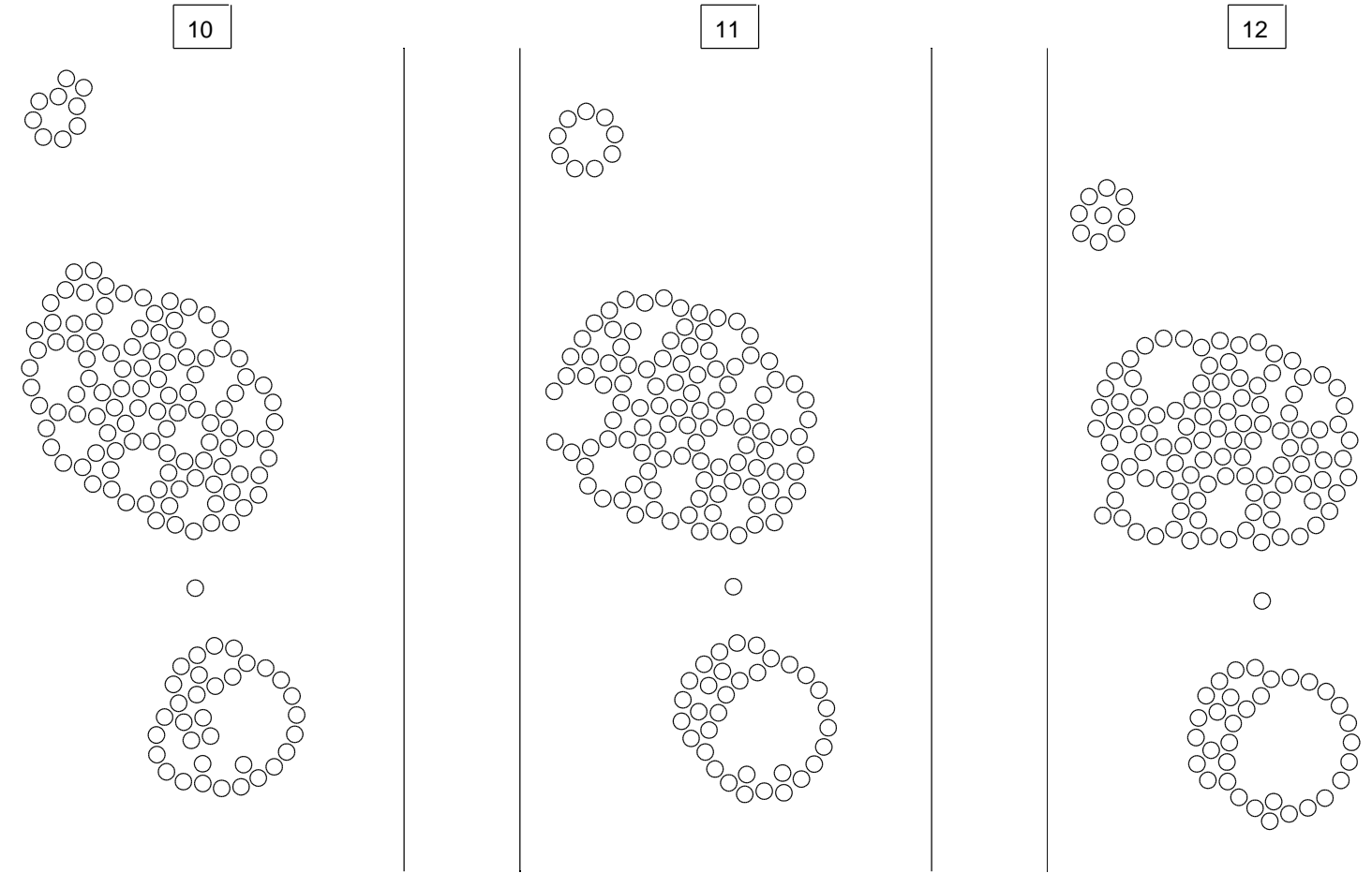

Figure 8: Configurations (7) to (12). Configurations (7) à (12).

\section{References}

[1] J.L. Amorós, V. Sanz, A. Gozalbo, B. Beltrán, Viscosity of concentrated clay suspensions : effect of solids volume fraction, shear stress, and deflocculant content, British Ceramic Transaction 101, 2002, 5, pp. 185-193.

[2] G.K. Batchelor, J.T. Green, The hydrodynamic interaction of two small freely moving spheres in a linear flow field, J. Fluid Mech. 56, pp. 375-400 (1972).

[3] L. Berlyand, L. Borcea, A. Panchenko, Network Approximation for Effective Viscosity of Concentred Suspensions with Complex Geometry, SIAM Journal on Mathematical Analysis, 36, no. 5, pp 1580-1628, 2005.

[4] L.J. Durlofsky, J.F. Brady, Dynamic simulation of bounded suspensions of hydrodynamically interacting particles, Journal of Fluid Mechanics 200, pp. 39-67 (1989).

[5] A. Einstein, Eine neue Bestimmung der Moleküledimensionen, Ann Phys. Leipsig 1906, 19, 289-306.

[6] R. Glowinski, T.W. Pan, T.I. Hesla, D.D. Joseph, A distributed Lagrange multiplier/fictitious domain method for particulate flow Int. J. of Multiphase Flow 25, 1998, pp. 755-794. 
[7] R. Glowinski, Finite element methods for incompressible viscous flow, in Handbook of numerical analysis, IX, 3-1176, North-Holland, Amsterdam, 2003.

[8] S. Haber, H. Brenner, Hydrodynamic interaction of spherical particles in quadratic Stokes flows, Int. J. Multiphase Flow 25, 1999, pp. 1009-1032.

[9] H.H. Hu, Direct simulation of flows of solid-liquid mixtures, Int. J. of Multiphase Flow 22, 1996, pp. 335-352.

[10] A. A. Johnson, T. E. Tezduyar, Simulation of Multiple Spheres Falling in a Liquid-Filled Tube, Computer Methods in Applied Mechanics and Engineering 134, pp. 351-373, 1996.

[11] S. Kim S. and S. J. Karrila, Microhydrodynamics: Principles and Selected Applications, Butterworth-Heinemann, Boston, 1991.

[12] B. Maury, Fluid-particle shear flow, ESAIM, 2003, 37, no. 4, pp. 699-708.

[13] B. Maury, Direct simulations of 2D fluid-particle flows in biperiodic domains, Journal of Computational Physics 156, 1999, pp. 325-351.

[14] P.Singh, T.I. Hesla, D.D. Joseph, Distributed Lagrange multiplier method for particulate flows with collisions, Int. J. of Multiphase Flow 29, 2003, pp. 495-509. 